ISSN: 2277-3754

ISO 9001:2008 Certified

International Journal of Engineering and Innovative Technology (IJEIT)

Volume 10, Issue 12, June 2021

\title{
Finding Community Infection Spreading Factor's presence in a Community
}

\author{
Saptarshi Naskar ${ }^{1}$, Arup Kr. Goswami ${ }^{2}$, Samar Sen Sarma ${ }^{3}$ \\ ${ }^{1}$ Department of CSE, Sarsuna College, University of Calcutta, India. \\ ${ }^{2}$ Department of CSE, Vidyasagar College, University of Calcutta, India. \\ ${ }^{3}$ Retired Professor, Department of CSE, University of Calcutta, India.
}

Abstract - In all the countries, all the communities consist of people having certain attitudes and interests in common. The whole community can be mapped into a graph network. Every distinct individual can be assumed as a node of the constructed graph for the community. To declare a pandemic situation for any country, we first need to check whether the infection is spread throughout the Community or not. For this purpose we need to calculate the existence of cliques and the nodes of the maximal clique will be the infection spreading factors. In this work, we try to find out the presence of these spreading factors in the community graph network.

Index Terms - Graphic sequence, Clique, Maximal Clique, Community Graph, Spreader factor.

\section{INTRODUCTION}

When an infection is spread in a community, first of all somehow (by GPS tracking / any means) we need to represent the community into a Graph and obviously, the generated graph will be a dense mess graph; then there is a probability of having several Cliques in the constructed graph. Hence among all Cliques, there must be at least one Maximal Clique. Now, the Maximal Clique of that graph consists of some vertices, which can be treated as the Community Infection Spreading Factors/elements/subjects.

\section{TECHNICAL EXPLANATION OF THE CONCEPT}

A sequence $\xi=d_{1}, d_{2}, d_{3}, \ldots, d_{n}$ of the grade of graph $G$ if the vertices of $\mathrm{G}$ can be designated $\mathrm{V} 1, \mathrm{~V} 2, \mathrm{~V} 3 \ldots \ldots . \mathrm{Vn}$, etc., as the grade $\mathrm{Vi}=\mathrm{di} ; \forall \mathrm{i}$ [2]. For a graph $\mathrm{G}$, it is easy to determine a grade sequence of $\mathrm{G}[1,3]$.

\section{A. Preliminaries}

In this section, we will define certain words in relation to our article and also some necessary theorems which form the backbone of the suggested method.

A sequence $\xi=d_{1}, d_{2}, d_{3}, \ldots, d_{n}$ of non-negative integer is called to be a graphical sequence if it is present in a graph $\mathrm{G}$ with vertices with the degree of di and $G$ termed the realization of a total number of numerical elements $[1,3]$.

A sequence $\xi$ has property Ap iff there is a graph with a grade sequence $\xi$, in which the first p-vertices constitute a complete subsection[2,5]. Above related theorem provides a way to determine whether $\xi$ has Ap property since it is straightforward to determine whether $\xi^{p, p}$ or not it is graphical.
This may be used to build a p-vertices graph (where it exists) which can also easily be determined from a graph with a degree sequence $\xi[2,3]$.

Procedure explanation by using an example

Consider a sequence,

$\xi^{4,1}=6,5,4,4,4,4,4,1$

With $p=4, \mathrm{~d}_{1}=6$

and since $p-1=3 \leq \mathrm{d}_{1} \leq(n-1)$

$\Rightarrow 3 \leq 6 \leq 7$

$\therefore \xi^{4,2}=4,3,3,3,3,3,1$

Now, $\mathrm{d}_{1}=4$ since, $p-1=3 \leq \mathrm{d}_{1} \leq(n-1)$

$\Rightarrow 3 \leq 4 \leq 7$

$\therefore \xi^{4,3}=2,2,3,2,2,1$

Now, $\xi^{4,4}=1,2,2,2,1$

Now, $\xi^{4,4}$ is graphic.

When working backward, a graph with a degree sequence $\xi$ is easy to design, in which four vertices form a whole sub graph.

$\xi^{5,1}=6,5,4,4,4,4,4,1$

With $p=5, \mathrm{~d}_{1}=6$ and since $p-1=4 \leq \mathrm{d}_{1} \leq(n-1)$

$\Rightarrow 4 \leq 6 \leq 7$

$\therefore \xi^{5,2}=4,3,3,3,3,3,1$

Now, $\mathrm{d}_{1}=4$ since, $p-1=4 \leq \mathrm{d}_{1} \leq(n-1)$

$\Rightarrow 4 \leq 4 \leq 7$

$\therefore \xi^{5,3}=2,2,2,3,2,1$

Now, $\xi^{5,4}=1,1,3,2,1$

Now, $\xi^{5,5}=0,3,2,1$

Since, $\xi^{5,5}$ is not graphic We infer that for the given degree sequence $\xi$ ident, a maximum clique number cannot be 5 ; it must be 4 .

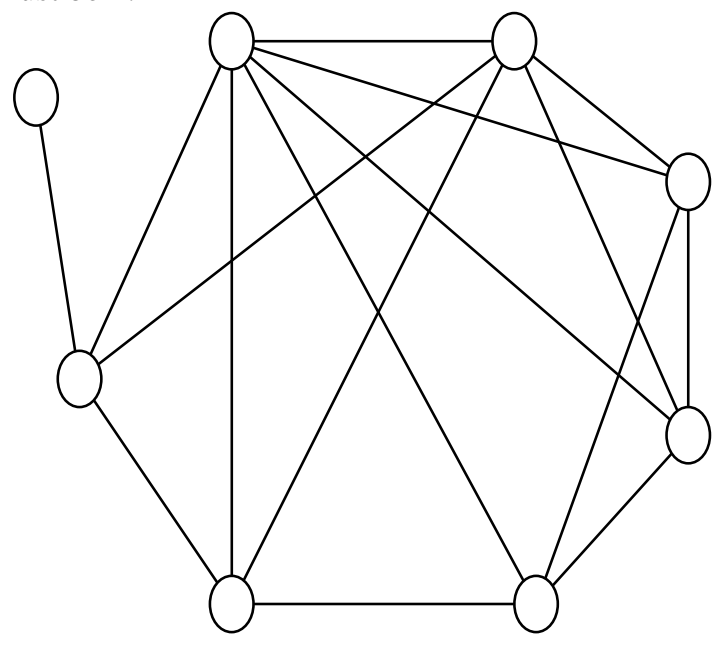

Fig-1.General Graph 
ISSN: 2277-3754

ISO 9001:2008 Certified

International Journal of Engineering and Innovative Technology (IJEIT)

\section{BASICS OF THE PROPOSED ALGORITHM}

The theorem on which the algorithm is based is presented below.

Theorem 2: Let $\xi=d_{1}, d_{2}, d_{3}, \ldots, d_{n}$ are the Grade sequence of $G$. Graph $\mathrm{G}$ comprises a maximum number of clicks $\mathrm{k}$ iff $\mathrm{d}_{1}$ $\geq k-1, k \leq n$ and graphic sequence after droop-m $\left(\mathrm{d}_{k+1}, \mathrm{~d}_{k+2}\right.$, $\left.\ldots, \mathrm{d}_{n}\right)$.

\section{A. Proposed algorithm}

Input: Non-negative integer sequence.

Output: K maximal number of the clique.

Step 1:

Step 2:

$$
\xi=\mathrm{d}_{1} \geq \mathrm{d}_{2} \geq \mathrm{d}_{3} \geq \ldots \geq \mathrm{d}_{n} \cdot k=\mathrm{d}_{1}
$$

If $\left(\mathrm{d}_{1} \geq \mathrm{d}_{2} \geq \mathrm{d}_{3} \geq \ldots \geq \mathrm{d}_{k} \geq(k-1)\right)$ go on;

Else

End If

Move to Step 4.

Step 3:

If $\left(\left(\mathrm{d}_{k+1}, \mathrm{~d}_{k+2}, \ldots, \mathrm{d}_{n}\right)\right.$ is graphical after drop- $\left.m\right)$ Then

Else Move to Step 5.

Go on.

End If

Step 4:

$$
\mathrm{k}=\mathrm{k}-1
$$

Step 5:

Print "The maximum clique number of the sequence $\xi$ is $k$ "

Step 6:

Quit.

\section{B. Description of the used technique with an example}

Let us consider a Grade sequence $\xi=6,6,4,4,4,4,2$, 2 and identify the maximal click number, indicating chart G. Now,

Since $\mathrm{d}_{1}=6$ therefore $k=\mathrm{d}_{1}=6$.

Now, $\mathrm{d}_{2} \geq 5$, but $\mathrm{d}_{3}<5$.

$$
\therefore k=k-1=5
$$

Now, $\mathrm{d}_{2} \geq \mathrm{d}_{3} \geq \ldots \geq \mathrm{d}_{5} \geq 4$

$\therefore m=\Sigma\left(\mathrm{d}_{i}-k+1\right) \forall i=1,2, . .5$.

$\therefore m=4$

$\therefore$ drop- $m$ from $\left(\mathrm{d}_{6}, \mathrm{~d}_{7}, \mathrm{~d}_{8}\right)$ we have

$$
\xi^{\prime}=(2,1,1)
$$

Which is graphic.

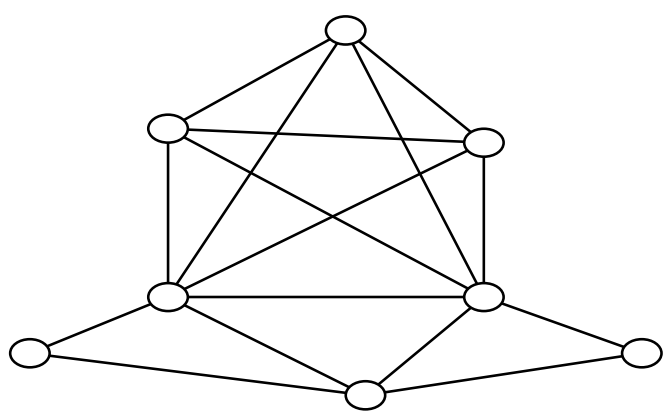

Fig.2 .The corresponding Clique of the sub-graph.

\section{CONCLUSION}

Identifying Maximal Clique from a Graph it can be identified required Community Infection Spreading Factors/elements/subjects and obviously it can be found in polynomial time and Govt. can take decision that whether Locked Down is required at that province or not.

\section{REFERENCES}

[1] Mario Coccia, "Factors determining the diffusion of COVID-19 and suggested strategy to prevent future accelerated viral infectivity similar to COVID”, Science of The Total Environment, Volume 729, August 2020, pp: 1-9.

[2] Yun Qiu, Xi Chen, Wei Shi, "Impacts of social and economic factors on the transmission of corona virus disease 2019 (COVID-19) in China", Journal of Population Economics, 2020, pp: 1127-1172.

[3] Arumugam S. and Ramachandran S., Invitation to Graph Theory, SciTech Publications (INDIA) Pvt. Ltd., Chennai, 2002 .

[4] N. Deo, Graph Theory with Applications to Engineering and Computer Science, Prentice-Hall of India Private Limited, New Delhi, 2003.

[5] Rao. A. R., Proceedings of the Symposium on Graph Theory held at ISI, Kolkata, ISI Lecture Notes, No. 4, 1976.

\section{AUTHOR BIOGRAPHY}

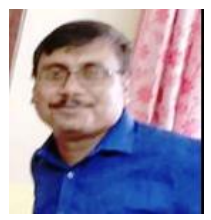

Saptarshi Naskar is presently working as Assistant Professor in Department of Computer Science, Sarsuna College, affiliated to University of Calcutta since 2008. His current research interest is in Combinatorics. $\mathrm{He}$ is presently pursuing $\mathrm{PhD}$ in Computer Science from University of Calcutta. He has 13 years of teaching experience and more than 13 years of research experience. He has more than 35 research articles in various peer reviewed journals.

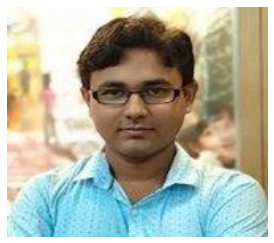

Arup Kr. Goswami pursued Bachelor of Science from Syamaprasad College, affiliated to University of Calcutta in 2015 and completed Master of Science (M.Sc) in Computer Science from Sarsuna College, affiliated to University of Calcutta in 2017. He is currently working in Department of Computer Science, Vidyasagar College, affiliated to University of Calcutta since 2019. His current research interest is in IoT. He has 3 years of teaching experience.

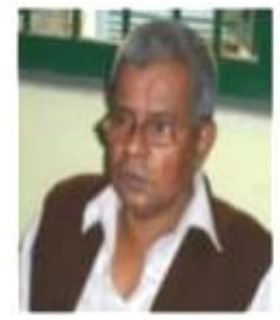

Dr. Samar Sen Sarma worked as the Professor in the Department of Computer Science and Engineering, University of Calcutta, India. He is one of the founder member of Department of CSE, University of Calcutta, India. He has published several papers in International journals and conferences. 